\title{
Efficacy of Dexamethasone in the Management of Malignant Small Bowel Obstruction in Advanced Epithelial Ovarian Cancer
}

\section{Antonia PM Jones}

Mercy Hospital for Women

Monica FG McGauran ( $D$ monica_fg_mcgauran@hotmail.com )

Mercy Hospital for Women https://orcid.org/0000-0002-2556-0755

\section{Nisha Jagasia}

Mater Hospital Brisbane

\section{Richard J Hiscock}

Mercy Hospital for Women

\section{Simon Hyde}

Mercy Hospital for Women

\section{Peter Grant}

Mercy Hospital for Women

\section{Research Article}

Keywords: Intestinal obstruction, Carcinoma, Ovarian Epithelial, Dexamethasone, Palliative care

Posted Date: August 12th, 2021

DOI: https://doi.org/10.21203/rs.3.rs-678458/v1

License: (1) This work is licensed under a Creative Commons Attribution 4.0 International License. Read Full License

Version of Record: A version of this preprint was published at Supportive Care in Cancer on November 30th, 2021. See the published version at https://doi.org/10.1007/s00520-021-06694-9. 


\section{Abstract}

Introduction: Malignant small bowel obstruction (MSBO) occurs in up to $50 \%$ of women with advanced epithelial ovarian cancer (EOC) causing symptom burden and distress to women and their families, particularly in the terminal stages of the disease. Corticosteroids are used to promote symptom resolution in malignant small bowel obstruction (MSBO) related to EOC, with little published data on their efficacy, optimal dosing and duration of treatment.

Objective: To evaluate the efficacy of dexamethasone in achieving symptom control in women with advanced EOC presenting with MSBO, assess dexamethasone dosing and efficacy over subsequent presentations, and examine differences in dexamethasone responsiveness between platinum resistant and platinum sensitive patient.

Methods: This is a retrospective cohort study of women presenting with MSBO due to advanced EOC over a 12-year period from January 2005 to December 2016 in a single tertiary hospital.

Results: Ninety-one women with MSBO were administered dexamethasone over 154 admissions with $89 \%$ of women initially achieving partial or complete symptom control. Dexamethasone responsiveness did not change with recurrent admissions and platinum responsive patients were more likely to respond to dexamethasone than platinum resistant patients (OR $3.6[95 \% \mathrm{Cl} 1.1$ to $12.2, \mathrm{p}=0.04]$ ). $15.6 \%$ of patients required additional measures to control symptoms of MSBO and $44.8 \%$ had adequate symptoms resolution to allow them to remain on or commence further treatment for EOC.

Conclusion: Dexamethasone therapy is a useful adjunctive therapy in the management of symptoms associated with MSBO in women with EOC.

\section{Introduction}

Ovarian cancer was the $17^{\text {th }}$ leading cause of death for females in Australia in 2019 with 1081 deaths reported[1]. In metastatic ovarian cancer, malignant bowel obstruction (MBO) has an estimated incidence of $20-51 \%$. [2-6] Median survival following MBO has been reported as 13-19 weeks.[3, 5-7] MBO is reported in up to $77 \%$ of women with advanced ovarian cancer.[7] Bowel obstruction in ovarian cancers more commonly involves the small bowel (61\% vs large bowel $33 \%$ ) with both sites involved in greater than $20 \%$ of patients. $[8,9]$ Malignant small bowel obstruction (MSBO) in advanced ovarian cancer is thought to be due to several factors: diffuse intra-peritoneal carcinomatosis infiltrating the bowel wall with oedema and accumulation of intramural cytokines leading to dysfunctional bowel motility; large volume deposits causing extrinsic or intramural occlusion from tumour growth; mesenteric and coeliac plexus deposits affecting the autonomic nerve supply; and adhesions preventing normal peristaltic action. $[10,11]$ Symptoms include colicky abdominal pain, anorexia, progressive nausea, vomiting, reflux, abdominal distention and cessation of flatus. Prolonged MSBO is complicated by malnutrition, dehydration and electrolyte abnormalities. MSBOs are distressing to women and their families and commonly require prolonged and recurrent inpatient hospital admissions during the terminal phase of 
their illness. Furthermore, acute MSBO can preclude the commencement of subsequent lines of cytotoxic therapies in eligible patients meaning MSBO needs to be managed to facilitate further therapy where indicated.

Historically, MSBO was treated with either nasogastric tube, intravenous fluid and electrolyte therapy or palliative surgery, often with inadequate management of the patient's symptoms. In 1985, a study of 38 patients in whom surgery was not a viable option suggested that management with corticosteroids shortened hospital length of stay and decreased the duration and severity of patient symptoms.[12]

Despite the common occurrence of MSBO in relapsed ovarian cancer there is an absence of level 1 evidence or national guidelines to aid management. Furthermore, there is no clear definition of "successful management" in MSBO. Despite attempts to establish clear criteria for randomised trials for malignant bowel obstruction, noting the importance of an observational arm and composite QOL outcome measures [13], a paucity of prospective, randomised trials means that most evidence comes from small case series, retrospective reviews and cohort studies, and management relies on clinicians' personal experience and individualisation of care.

A Cochrane review published in 2000 on the use of corticosteroids for the resolution of MBO in advanced gynaecological and gastrointestinal cancer identified very limited data but suggested a potential benefit, with a trend towards faster resolution of bowel obstruction using corticosteroids. Although limited by data quality, the morbidity from corticosteroid use appeared to be very low. It should be noted that the Cochrane review used a heterogeneous population with different histological subtypes and no distinction regarding the site of obstruction. [14]

Corticosteroid actions are multifactorial and not completely understood. Corticosteroids are thought to provide anti-emetic and co-analgesic effects and to reduce peri-tumour and peri-neural inflammatory oedema, improving intestinal motility. There is uncertainty about their efficacy, the optimal choice of steroid, route of administration, dosing regime and duration of treatment.[14] Short term side effects from steroid administration include delirium, sleep disturbance, proximal muscle weakness, gastric ulceration, infection risk and worsening of glycaemic control in diabetic patients.

We present a retrospective cohort study of the use of one specific corticosteroid (dexamethasone) for MSBO at our centre. This study aims to further inform evidence regarding the effectiveness of dexamethasone for MSBO in advanced EOC. It also aims to inform evidence regarding the duration and dose of corticosteroid and response.

\section{Materials And Methods}

This is a retrospective cohort study conducted at the Mercy Hospital for Women, a tertiary women's hospital in Heidelberg, Victoria, Australia, caring for approximately 55 women per year with newly diagnosed with ovarian cancer. The study included all women treated with dexamethasone who had a history of epithelial ovarian cancer (all histopathological subtypes and stages) with advanced disease 
and were admitted with a MSBO or combined small and large bowel obstruction between 1 January 2005 and 1 December 2016.

The primary outcome was to evaluate the efficacy of dexamethasone in achieving full or partial symptoms control in patients with advanced epithelial ovarian cancer presenting with MSBO. The secondary outcomes were: (1) to evaluate whether the efficacy of dexamethasone declines during subsequent admissions with recurrent MSBO; (2) to compare dexamethasone dosage required to achieve symptom control in the first versus recurrent episodes of MSBO; (3) to compare the efficacy of dexamethasone in chemotherapy refractory, platinum resistant and platinum sensitive disease; and (4) to assess the prognostic significance of MSBO in patients with advanced ovarian cancer.

Data was obtained from review of the patient medical record. The diagnosis of bowel obstruction was based upon a patient's signs, symptoms and imaging during admission (abdominal X-ray or Computerized Tomography (CT) of the abdomen pelvis). A diagnosis of partial bowel obstruction was made when there was ongoing passage of flatus and liquid stool, whereas complete bowel obstruction was diagnosed in the setting of failure to pass flatus and liquid stool and/or an empty rectum on radiography. The medical record was reviewed to determine patient demographics including age, 2014 International Federation of Gynaecology and Obstetrics (FIGO) stage at diagnosis, histopathological subtype, site of primary disease, date of diagnosis, date of death; primary treatment; recurrence data; number of presentations with MSBO; MSBO symptoms, dexamethasone dosage and duration; alternate management (including surgery); resolution of MSBO with symptom control and clinical outcome.

Complete symptom control was defined as discharge (to home or other, including palliative care facility) on full diet with resolution of all symptoms. Partial symptom control was defined as symptom improvement as reported by the patient and discharge tolerating clear or free fluids and oral medication with possible mild persistent symptoms such as nausea and or vomiting.

Conservative management of MSBO included nil by mouth (ice for comfort), intravenous fluids, electrolyte optimisation, analgesics, antiemetics and anti-reflux medication (usually a proton pump inhibitor). Other interventions in selected cases only included the use of a nasogastric tube (NGT), gastrograffin enema, percutaneous endoscopic gastrostomy (PEG) tube, ascitic tap or surgery. Usual dose of Dexamethasone was $8 \mathrm{mg} /$ day with a reducing dose of $4 \mathrm{mg} /$ day following resolution of symptoms.

Platinum resistant disease was defined as evidence of progressive disease (radiological, clinical or biochemical) within six months of completing first line platinum based chemotherapy.

Women who had evidence of isolated large bowel obstruction (where surgery is more often required) and women who had no evidence of advanced ovarian cancer as a cause of their obstruction were excluded, as were women with MSBO occurring during primary therapy (either perioperatively or during first line chemotherapy). Analysis was truncated for the first six admissions with MSBO. 
Patient characteristics and outcome data were summarized as mean (SD), median [25th to 75th percentile] and number (\%) according to type and distribution. For outcomes the precision of percentage point estimates was presented as $95 \%$ confidence intervals calculated using the Wilson score test. Assessment of longitudinal change across presentations for responsiveness and dosage per was performed using a marginal mean model using generalized estimation equations with robust standard errors and an exchangeable correlation structure. All statistical tests were two-sided, with a p value of < 0.05 considered significant. Statistical analysis was performed using Stata v16 statistical software (StataCorp. 2019.Stata Statistical Software: Release 16. College Station, T. USA).

Ethics approval was obtained through the Mercy Health Human Research Ethics Committee (Reference number R16/50, approved 11 October 2016).

\section{Results}

One-hundred and fifty-four admissions for 91 patients met inclusion criteria (Fig. 1). Baseline characteristics are displayed in Table 1. 76.9\% of patients were diagnosed with FIGO stage 3 disease and $16.5 \%$ with FIGO stage 4 disease. The median interval from cancer diagnosis to the first presentation with MSBO was 22.9 months [IQR 11.1-46 months]. The median interval from diagnosis of cancer recurrence to MSBO was 10.9 months (IQR 2.8-25.0 months). The median number of admissions with MSBO was one [IQR 1-2], however $25 \%$ of women had three or more admissions for MSBO. 
Table 1

Baseline characteristics $(\mathrm{N}=91)$

\begin{tabular}{|ll|}
\hline Age at cancer diagnosis [mean (SD)] & $59(12)$ \\
\hline Age at first MSBO [mean (SD)] & $62(11)$ \\
\hline FIGO ${ }^{\$}$ stage at diagnosis [n (\%)] & $1(1 \%)$ \\
\hline C & $1(1 \%)$ \\
\hline 2a & $2(2 \%)$ \\
\hline 2b & $1(1 \%)$ \\
\hline $3 a$ & $7(8 \%)$ \\
\hline $3 b$ & $62(68 \%)$ \\
\hline $3 c$ & $6(7 \%)$ \\
\hline $4 a$ & $9(10 \%)$ \\
\hline $4 b$ & $2(2 \%)$ \\
\hline Not documented & $7(1 \%)$ \\
\hline Histology [number (\%)] & $70(77 \%)$ \\
\hline Serous adenocarcinoma (presumed high grade) & $1(1 \%)$ \\
\hline Low grade serous adenocarcinoma & $6(7 \%)$ \\
\hline Clear cell adenocarcinoma & $3(3 \%)$ \\
\hline Adenocarcinoma, favour female genital tract origin & $3(3 \%)$ \\
\hline Endometrioid adenocarcinoma & $2(2 \%)$ \\
\hline Mucinous adenocarcinoma & $41(45 \%)$ \\
\hline Undifferentiated carcinoma & \\
\hline Mixed (serous and clear cell carcinoma) & $1(1 \%)$ \\
\hline Mixed (endometrioid and clear cell carcinoma) & \\
\hline Platinum resistant disease [number (\%)] & \\
\hline
\end{tabular}

Legend: *MSBO - Malignant small bowel obstruction; \$ FIGO - International Federation of Gynaecology and Obstetrics ; SD - Standard deviation

The presenting symptoms are described in Table 2. Ninety three percent of all presentations were due to partial small bowel obstruction and $7 \%$ to complete small bowel obstruction (Table 3 ). 
Table 2

Documented Symptoms of patients presenting with mechanical small bowel obstruction across all presentations.

\begin{tabular}{|ll|}
\hline Documented Symptoms & Symptom reported during admission (\%) ( $\mathbf{n}=179)$ \\
\hline Nausea & $155(86.6 \%)$ \\
\hline Vomiting & $146(81.6 \%)$ \\
\hline Abdominal Pain & $126(70 \%)$ \\
\hline Loss of appetite & $37(20.7 \%)$ \\
\hline Bowel & \\
\hline - Constipation & $106(59.2 \%)$ \\
\hline - Diarrhoea & $21(11.7 \%)$ \\
\hline
\end{tabular}

Table 3

Complete vs partial bowel obstruction

\begin{tabular}{|c|c|c|c|c|c|c|}
\hline & \multicolumn{6}{|c|}{ Admission } \\
\hline & $1 \mathrm{st}$ & 2nd & $3 r d$ & 4th & 5 th & 6th \\
\hline & $\begin{array}{l}(n= \\
91)\end{array}$ & $\begin{array}{l}(n= \\
44)\end{array}$ & $\begin{array}{l}(n= \\
22)\end{array}$ & $\begin{array}{l}(n= \\
12)\end{array}$ & $\begin{array}{l}(n= \\
8)\end{array}$ & $(n=2)$ \\
\hline \multicolumn{7}{|l|}{ Small Bowel obstruction } \\
\hline Partial & $\begin{array}{l}86 \\
(95 \%)\end{array}$ & $\begin{array}{l}41 \\
(93 \%)\end{array}$ & $\begin{array}{l}20 \\
(91 \%)\end{array}$ & $\begin{array}{l}11 \\
(92 \%)\end{array}$ & $\begin{array}{l}7 \\
(88 \%)\end{array}$ & $\begin{array}{l}2 \\
(100 \%\end{array}$ \\
\hline Complete & $5(5 \%)$ & $3(7 \%)$ & $2(9 \%)$ & $1(8 \%)$ & $\begin{array}{l}1 \\
(12 \%)\end{array}$ & 0 \\
\hline $\begin{array}{l}\text { Combined Small and Large Bowel } \\
\text { obstruction }\end{array}$ & $4(4 \%)$ & $1(2 \%)$ & 0 & 0 & 0 & 0 \\
\hline
\end{tabular}

\section{Primary Outcome}

The details of dexamethasone responsiveness and dosing are presented in Table 4. Overall, 137 (89\%) admissions were associated with a complete or partial response to dexamethasone. 
Table 4

Dexamethasone dose and responsiveness

Presentation

\begin{tabular}{lllllll} 
(Dexamethasone administered) & $1 \mathrm{st}$ & $2 \mathrm{nd}$ & $3 \mathrm{rd}$ & 4 th & 5 th & 6th \\
& $(\mathrm{n}=75)$ & $(\mathrm{n}=42)$ & $(\mathrm{n}=18)$ & $(\mathrm{n}=10)$ & $(\mathrm{n}=7)$ & $(\mathrm{n}=2)$ \\
\hline $\begin{array}{l}\text { Response to dexamethasone } \\
\text { (complete or partial) }\end{array}$ & 70 & 37 & 14 & 9 & 5 & 2 \\
& $(93.3 \%)$ & $(88.0 \%)$ & $(77.8 \%)$ & $(90.0 \%)$ & $(71.4 \%)$ & $(100 \%)$
\end{tabular}

(\%)

\begin{tabular}{|c|c|c|c|c|c|c|}
\hline $\begin{array}{l}\text { Median duration of } \\
\text { dexamethasone (days) (IQR) }\end{array}$ & $5(4-7)$ & $5(3-8)$ & $\begin{array}{l}6(4- \\
11)\end{array}$ & $4(3-5)$ & $6(5-6)$ & 6 \\
\hline $\begin{array}{l}\text { Mean\$ dosage per day (mg) } \\
\text { [median (IQR)] }\end{array}$ & $6(4-8)$ & $7(5-8)$ & $8(5-8)$ & $6(4-8)$ & $7(4-8)$ & 11 \\
\hline $\begin{array}{l}\text { Total dose given (mg) } \\
\text { [median (IQR)] }\end{array}$ & $\begin{array}{l}40(26- \\
56)\end{array}$ & $\begin{array}{l}32(21- \\
56)\end{array}$ & $\begin{array}{l}32(21- \\
84)\end{array}$ & $\begin{array}{l}26 \\
(16- \\
35)\end{array}$ & $\begin{array}{l}40 \\
(26- \\
40)\end{array}$ & 38 \\
\hline \multicolumn{7}{|c|}{ Dexamethasone responsiveness of platinum sensitive and platinum resistant patients } \\
\hline Platinum resistant & $\begin{array}{l}32 / 36 \\
(88.6 \%)\end{array}$ & $\begin{array}{l}15 / 19 \\
(78.9 \%)\end{array}$ & $\begin{array}{l}9 / 11 \\
(81.8 \%)\end{array}$ & $\begin{array}{l}6 / 7 \\
(85.7 \%)\end{array}$ & $\begin{array}{l}2 / 4 \\
(50 \%)\end{array}$ & 0 \\
\hline Platinum sensitive & $\begin{array}{l}38 / 39 \\
(97.4 \%)\end{array}$ & $\begin{array}{l}22 / 23 \\
(95.7 \%)\end{array}$ & $\begin{array}{l}5 / 7 \\
(71.4 \%)\end{array}$ & $\begin{array}{l}3 / 3 \\
(100 \%)\end{array}$ & $\begin{array}{l}3 / 3 \\
(100 \%)\end{array}$ & $\begin{array}{l}2 / 2 \\
(100 \%)\end{array}$ \\
\hline
\end{tabular}

\section{Secondary outcomes}

Based upon the longitudinal regression model there was no statistically significant difference in mean responsiveness over repeated admissions $(p=0.14)$, the predicted mean responsiveness for any admission was $89 \%$ (95\% $\mathrm{Cl} 84$ to 94$)$.

The median duration of dexamethasone dosing was 4 to 6 days, and the median daily dose was between 6-8 milligrams. The median total dose was between 26 and $40 \mathrm{mg}$. Doses were not statistically different across admissions $(p=0.96)$ with predicted mean daily dose of $6.5 \mathrm{mg}(95 \% \mathrm{Cl} 6.0$ to 7.0$)$.

Platinum sensitive patients were more likely to respond to dexamethasone compared to platinum resistant patients (OR 3.6 [95\% $\mathrm{Cl} 1.1$ to $12.2, \mathrm{p}=0.04]$ ) (Table 4). In addition, there was no statistical difference in dexamethasone responsiveness between first and subsequent presentations for either platinum sensitive or resistant patients $(p=0.11)$.

The median length of stay ranged from 5 to 7 days and was not statistically different between presentations. 
Across all admissions, additional treatments were used to aid in resolution of symptoms in $16 \%$ of cases, notably $6 \%$ needed an ascitic tap, $6 \%$ needed NGT placement and $3 \%$ required an exploratory laparotomy, adhesiolysis and bowel resection / bypass procedure and / or loop ileostomy.

Regarding management after presentation with MSBO, $38 \%$ of women received end-of-life care, $6 \%$ were observed but $45 \%$ remained on disease treatment (continued or commenced chemotherapy, clinical trial, hormonal therapy or radiotherapy). The median time from first presentation with MSBO to death was 3.8 months (IQR 1.9-9.8).

\section{Discussion}

\section{Key findings}

This retrospective cohort study demonstrates a complete or partial response rate of $89 \%$ in patients administered dexamethasone when presenting with MSBO. There was no difference in response to treatment over multiple presentations with MSBO. In addition, we have shown that platinum sensitive patients are more likely to have symptom control than platinum resistant patients after treatment for MSBO. Patients required additional therapies to resolve their MSBO relatively infrequently. Median duration of therapy was 4 to 6 days and median daily dose 6 to $8 \mathrm{mg}$. This study confirms that short term mortality is high following first presentation with a MSBO associated with recurrent disease with a median survival of 3.8 months.

\section{Comparison to previous studies}

Our retrospective review is one of the largest data sets of women with advanced EOC and MSBO to examine the efficacy of dexamethasone. Complete or partial resolution of symptoms after receiving dexamethasone for treatment of MSBO is higher in our study that previously reported. In a study of 35 patients with MBO treated with dexamethasone versus placebo, Hardy et al. found resolution rates of $37 \%$ vs $22 \%$ respectively.[15] A prospective study by Philip et al of 13 patients found $69 \%$ response to a three day minimum course of dexamethasone.[16] Laval et al studied 52 patients treated with methylprednisolone versus placebo and showed a non-statistically significant trend toward higher symptom resolution (59\% versus 33.5\%).[17] The low resolution of symptoms in these studies may be because treatment success or failure was assigned on day four of treatment in the in the study by Laval et al [16], as well as the use of methylprednisolone rather than dexamethasone and the smaller patient numbers in both studies. The inclusion of bowel obstruction at all levels (gastric outlet, small and large bowel) may also account for differences in results.

Use of other therapies in our study is lower than that reported in other studies. Tran et al found that amongst 49 patients with MBO, $42.9 \%$ required surgery and $14.3 \%$ required other interventions, such as stent placement.[4] A Surveillance, Epidemiology, and End Results registry (SEER) database review of 1,397 women who were admitted with bowel obstruction more than 6 months post stage II-IV ovarian cancer diagnosis showed that $40 \%$ underwent surgery and $11 \%$ had a gastrostomy or jejunostomy tube 
placed.[18] Explanations for this could be that the SEER database review did not limit patients to those with malignant bowel obstruction secondary to advanced disease and so surgical intervention for other indications such as incarcerated hernias and adhesions would have been included.

Our study confirms numerous previous findings of poor median survival after presentation with MSBO.[3, 5-7] Tran et al. described a cohort of 311 patients with recurrent ovarian cancer, of which 49 had bowel obstruction after recurrence. Of these, 29 lived less than 6 months after their bowel obstruction.[4]

\section{Implications of our study findings}

Our study population is representative of the Australian population due to a broad referral base. Our results support the use of Dexamethasone in the supportive treatment of MSBO in advanced EOC. The results can be used to counsel women who present with a MSBO in the context of advanced EOC. Importantly, given our study supports previous data that shows median survival is short, priority should be given to helping women develop an advanced care plan in consultation with their support people and treating practitioners. Despite poor long-term survival, over $40 \%$ of women remained on or re-commenced treatment. As this is a retrospective review, it is unclear the extent to which counselling, acknowledgment of treatment goals and shared decision making went into such treatment decisions for these women.

It must be highlighted that all management of women with MSBO must be regarded as palliative and, in general, avoidance of surgery is advisable, as any intervention (medical/surgical/venting decompression) that does not facilitate further chemotherapy does not appear to be associated with an improved survival and should be considered palliative.[8, 18, 19]

\section{Future directions}

An interprofessional MBO program incorporating non-surgical management (including pharmacological therapy) and prioritising an outpatient model of care would provide an organised framework to coordinate the complex care needs of a patient between multiple disciplines and so optimise her experience. A pilot program was introduced in a large Canadian cancer centre and resulted in a statistically significantly shorter length of stay in hospital (13 vs 22 days) and an improved median overall survival (99 vs 243 days) adjusted for stage and lines of chemotherapy. [2] Important components included patient educational material for patient empowerment, specialised oncology nurses leading telephone consultations in between clinic appointments, multidisciplinary team review of the cases and treatment consensus based on standardized clinical assessment tools and finally, electronic documentation to facilitate timely in and outpatient care. It is possible that incorporating the early use of corticosteroids for MSBO, in carefully supervised outpatient setting, for patients being palliated for advanced ovarian cancer, may further improve such programs.

\section{Strengths and limitations}

This study is one of the larger data sets of women with advanced EOC and MSBO to be published, however its limitations stem from the fact it is a retrospective, single centre observational review. No data on possible adverse effects from corticosteroid administration or the patient's experience from taking 
corticosteroids could be collected, but the morbidity from steroid administration appears to be low in this setting. [20] Patients who didn't receive dexamethasone for an admission were not included in analysis, limiting the ability to perform a comparative review of effectiveness of dexamethasone versus no dexamethasone. Given dexamethasone is standard of care in this unit, those who did not receive this treatment were selected because they had mild or resolving symptoms and therefore due to this selection bias no comparison was performed.

\section{Conclusion}

Dexamethasone therapy achieved $89 \%$ complete or partial symptom control in women who have advanced EOC presenting with MSBO, whose life expectancy is under four months after first presentation. Duration of therapy was 4 to 6 days with a 6 to $8 \mathrm{mg}$ median daily dose. Quality of life is critical in any palliative intent treatment and patient preferences should be evaluated in future studies assessing patient centred outcomes and patient reported experience measures from the management of their bowel obstruction, including steroid use and its possible side effects and their place in the management of a bowel obstruction. Adequate control of symptoms should be defined by the patient group and national guidelines. Incorporating multidisciplinary MBO care will likely improve patient experience at the end of their lives.

\section{Declarations}

Funding: The authors did not receive funding from any organisation for the submitted work.

Conflict of Interest/Competing Interest: The authors have no conflict of interest to declare. Availability of data and material: Data was obtained from the patients' medical record.

Code availability: Statistical analysis was performed using Stata v16 statistical software (StataCorp. 2019. Stata Statistical Software: Release 16. College Station, T. USA)

Author contributions: All authors contributed to the study conception and design. Data collection performed by AJ, MM and NJ. Analysis was performed by RH. The first draft of the manuscript was written by $\mathrm{AJ}$ and all authors commented on previous versions of the manuscript. All authors read and approved the final manuscript.

Ethics approval: Ethics approval was obtained from the Mercy Health Human Research Ethics Committee. (Reference number R16/50, approved 11 October 2016).

Consent to participate: As retrospective data was obtained from the medical record, the need to obtain consent to participate was waived by the Mercy Health HREC.

Consent for publication: N/A 


\section{References}

1. Australian Bureau of Statistics. (2018) Causes of Death, Australia. https://www.abs.gov.au/statistics/health/causes-death/causes-death-australia/2018. Accessed 4 January 2021

2. Lee YC, Jivraj N, Wang L, Chawla T, Lau J, et al. (2019) Optimizing the Care of Malignant Bowel Obstruction in Patients With Advanced Gynecologic Cancer. Journal of Oncology Practice 0: JOP.18.00793.

3. Mooney SJ, Winner M, Hershman DL, Wright JD, Feingold DL, et al. (2013) Bowel obstruction in elderly ovarian cancer patients: a population-based study. Gynecol Oncol 129: 107-12.

4. Tran E, Spiceland C, Sandhu NP, Jatoi A (2016) Malignant Bowel Obstruction in Patients With Recurrent Ovarian Cancer. Am J Hosp Palliat Care 33: 272-5.

5. Sartori E, Chiudinelli F, Pasinetti B, Maggino T (2009) Bowel obstruction and survival in patients with advanced ovarian cancer: analysis of prognostic variables. Int J Gynecol Cancer 19: 54-7.

6. Sartori E, Chiudinelli F, Pasinetti B, Zanagnolo V (2005) Palliative care in advanced ovarian cancer patients with bowel obstruction. Gynecol Oncol 99: S215-6.

7. Martinez Castro P, Vargas L, Mancheno A, Martin Utrilla S, Pascual F, et al. (2017) Malignant Bowel Obstruction in Relapsed Ovarian Cancer With Peritoneal Carcinomatosis: An Occlusive State. Int J Gynecol Cancer 27: 1367-1372.

8. Daniele A, Ferrero A, Fuso L, Mineccia M, Porcellana V, et al. (2015) Palliative care in patients with ovarian cancer and bowel obstruction. Support Care Cancer 23: 3157-63.

9. Caceres A, Zhou Q, lasonos A, Gerdes H, Chi DS, et al. (2008) Colorectal stents for palliation of largebowel obstructions in recurrent gynecologic cancer: an updated series. Gynecol Oncol 108: 482-5.

10. Bais J, Schilthuis M, Ansink A (2002) Palliative management of intestinal obstruction in patients with advanced gynaecological cancer. CME Journal of Gynecologic Oncology 7: 299-305.

11. Cousins SE, Tempest E, Feuer DJ (2016) Surgery for the resolution of symptoms in malignant bowel obstruction in advanced gynaecological and gastrointestinal cancer. Cochrane Database Syst Rev: Cd002764.

12. Baines M, Oliver DJ, Carter RL (1985) Medical management of intestinal obstruction in patients with advanced malignant disease. A clinical and pathological study. Lancet 2: 990-3.

13. Anthony T, Baron T, Mercadante S, Green S, Chi D, et al. (2007) Report of the clinical protocol committee: development of randomized trials for malignant bowel obstruction. J Pain Symptom Manage 34: S49-59.

14. Feuer DJ and Broadley KE (2000) Corticosteroids for the resolution of malignant bowel obstruction in advanced gynaecological and gastrointestinal cancer. Cochrane Database Syst Rev: Cd001219.

15. Hardy J, Ling J, Mansi J, Isaacs R, Bliss J, et al. (1998) Pitfalls in placebo-controlled trials in palliative care: dexamethasone for the palliation of malignant bowel obstruction. Palliat Med 12: 437-42. 
16. Philip J, Lickiss N, Grant PT, Hacker NF (1999) Corticosteroids in the management of bowel obstruction on a gynecological oncology unit. Gynecol Oncol 74: 68-73.

17. Laval G, Girardier J, Lassaunière JM, Leduc B, Haond C, et al. (2000) The use of steroids in the management of inoperable intestinal obstruction in terminal cancer patients: do they remove the obstruction? Palliat Med 14: 3-10.

18. Suidan RS, He W, Sun CC, Zhao H, Ramondetta LM, et al. (2017) Treatment Patterns, Outcomes, and Costs for Bowel Obstruction in Ovarian Cancer. Int J Gynecol Cancer 27: 1350-1359.

19. Dean E, Khoja L, Clamp A, Jayson GC, Goonetilleke D, et al. (2017) Malignant bowel obstruction in advanced ovarian cancer. Future Oncol 13: 513-521.

20. Feuer DJ and Broadley KE (1999) Systematic review and meta-analysis of corticosteroids for the resolution of malignant bowel obstruction in advanced gynaecological and gastrointestinal cancers. Systematic Review Steering Committee. Ann Oncol 10: 1035-41.

\section{Figures}




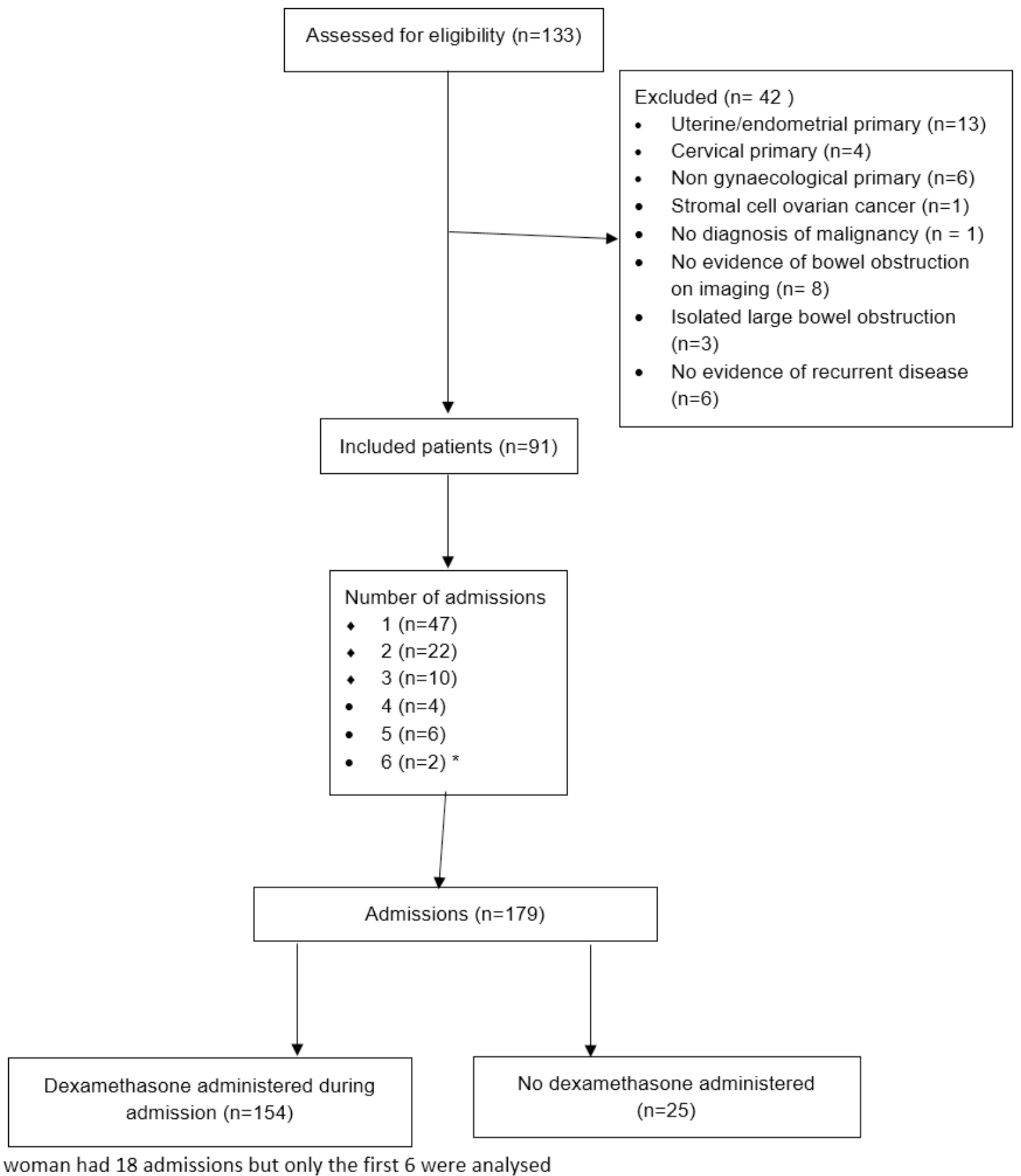

* 1 woman had 18 admissions but only the first 6 were analysed

\section{Figure 1}

CONSORT diagram 